\title{
Acute Colonic Pseudoobstruction
}

\author{
Allen P. Chudzinski, MD ${ }^{1}$ Earl V. Thompson, MD ${ }^{2}$ Jennifer M. Ayscue, MD ${ }^{1,3}$ \\ ${ }^{1}$ Colorectal Surgery Program, Department of Surgery, MedStar \\ Georgetown University Hospital, Washington, District of Columbia \\ ${ }^{2}$ Department of Surgery, MedStar Washington Hospital Center, \\ Washington, District of Columbia \\ ${ }^{3}$ Section of Colon and Rectal Surgery, Washington Hospital Center, \\ Washington, District of Columbia \\ Address for correspondence Jennifer M. Ayscue, MD, 106 Irving \\ Street, NW, Suite 2100 North, Washington, DC 20010 \\ (e-mail: jennifer.m.ayscue@medstar.net). \\ Clin Colon Rectal Surg 2015;28:112-117.
}

\begin{abstract}
Keywords

- colonic ileus

- acute colonic pseudoobstruction

- Ogilvie syndrome

- large bowel obstruction

Acute colonic pseudoobstruction (ACPO), often referred to as Ogilvie syndrome, is a clinical entity characterized by severe colonic distension (adult acute megacolon) in the absence of mechanical obstruction. It can result in abdominal ischemia and perforation if left untreated. This article discusses the epidemiology and current pathophysiologic theories of ACPO as well as the clinical presentation and diagnostic modalities utilized to identify the disease. In addition, this article describes the current treatment options for ACPO, which range from conservative medical therapy, therapeutic endoscopy, to subtotal colectomy.
\end{abstract}

Acute colonic pseudoobstruction (ACPO) is a clinical entity characterized by severe colonic distension in the absence of mechanical obstruction. It is often referred to by its historical eponym, Ogilvie syndrome, but this terminology has fallen out of favor as insufficiently precise. Patients are most often elderly, afflicted by multiple comorbidities, nursing home residents, or hospitalized after recent nonabdominal surgery. Patients suffering from progressive neurologic diseases, such as Parkinson or Alzheimer, fall victim to ACPO at a higher rate. The exact pathogenesis of this condition has not been completely described but it is thought to result from an imbalance of the enteric nervous system as a result of acute illness within the background of chronic disease.

Because the distension of the colon can become massive enough to cause ischemia and perforation, early diagnosis and treatment are critical for patient survival. When considering a patient population suffering from dementia or other neurologic deficit which impairs communication, the clinician must have a high clinical suspicion to arrive at the correct diagnosis. Treatment for ACPO has evolved considerably in recent years, and an algorithm-based approach centering on early pharmacologic treatment has become widely accepted.

\section{Epidemiology, Etiology, and Associated Conditions}

As ACPO is a sporadic and often underreported disease, there is an incomplete understanding of its incidence. However, the typical patient is described as elderly with multiple chronic diseases. Often, the patient is hospitalized or a resident of a long-term care facility. Published case series have documented mean ages of 64 to 74 years. ${ }^{1,2}$

ACPO has been associated with a diverse array of underlying conditions. These include both acute physiologic insults, such as abdominal and nonabdominal surgery which can contribute to colonic ileus, along with chronic diseases, such as systemic lupus erythematosus and hematologic malignancies. In a series of 1,027 patients, Wegener et al found ACPO to be most commonly associated with postoperative conditions (23\%) followed by cardiopulmonary disorders (17.5\%), other systemic disorders (15\%), and trauma $(11 \%)^{2}$ While there are reports of ACPO associated with countless diseases and conditions, the most common are included in - Table 1.
Issue Theme Miscellaneous Colitides: What the Colorectal Surgeon Needs to Know; Guest Editor: Anjali S. Kumar, $M D, M P H, F A C S$, FASCRS
Copyright @ 2015 by Thieme Medical Publishers, Inc., 333 Seventh Avenue, New York, NY 10001, USA. Tel: +1(212) 584-4662.
DOI http://dx.doi.org/ 10.1055/s-0035-1549100. ISSN 1531-0043. 
Table 1 Conditions associated with acute colonic pseudoobstruction

\begin{tabular}{|l|l|}
\hline Category & Inciting event or causative agent \\
\hline Surgical & Coronary bypass, solid organ transplantation, major orthopedic (hip or knee replacement), spine surgery \\
\hline Cardiac & Shock, myocardial infarction, congestive heart failure \\
\hline Neurologic & Dementia, Parkinson disease, stroke, spinal cord injury \\
\hline Metabolic & Electrolyte imbalance, diabetes, renal failure \\
\hline Medications & Immunosuppressive, chemotherapeutic, opiate, antiparkinson, clonidine \\
\hline Oncologic & Allogenic stem cell transplantation, pediatric hematologic malignancies \\
\hline Obstetric & Cesarean section, normal vaginal delivery, normal pregnancy \\
\hline Infectious & Varicella-zoster virus, herpes virus, cytomegalovirus \\
\hline Miscellaneous & Major burn/trauma, systemic lupus erythematosus, sickle cell disease \\
\hline
\end{tabular}

\section{Pathophysiology}

The exact pathogenesis of ACPO is yet to be completely described. In 1948, the British surgeon Sir William Heneage Ogilvie published an original report of two patients with large tumors in the region of the esophageal hiatus whose symptoms of colonic obstruction had no mechanical cause. The clinical syndrome is attributed to unopposed parasympathetic activity after the sympathetic supply was disrupted by the tumor. $^{3}$ Sympathetic innervation to the proximal colon is provided by the splanchnic nerves, while the vagus nerves supply parasympathetic input. Recent investigations into the pathophysiology of gut motility disorders have increasingly focused on the various neurotransmitters that mediate enteric nervous system control. Acetylcholine (Ach), neurokinin $A$, and substance $P$ are the predominate stimulatory neurotransmitters while vasoactive intestinal peptide and nitric oxide (NO) are inhibitory neurotransmitters. ${ }^{4}$ In a recent study of rats who have undergone hypergravity-stress conditioning, analogous to the physiologic stress of a human with chronic disease, colonic wall contractility is significantly potentiated in response to neurotransmitters. Sections of colon from stress-conditioned rats show greater contractility when treated with Ach and greater antagonization when treated with adrenaline, compared with controls or ileal sections. ${ }^{5}$ Rats undergoing physiologic stress in a model of distal colitis suffer decreased contractility and dilation of the proximal untreated colon, which is reversed by inhibitors of NO synthase. ${ }^{6}$ In the proximal colon of normal guinea pigs, Ach-mediated contraction is followed by NO-mediated relaxation. However, Ach stimulates contraction without relaxation in the middle part of the colon. ${ }^{7}$ Taken together, these findings suggest a mechanism for cecal dilatation often noted in ACPO as potentiated Ach stimulation leads to transient contraction followed by NO-mediated decreased contractility and dilatation of the proximal colon. Thus, the dilatation seen in ACPO is generally proximal to the splenic flexure.

\section{Clinical Presentation}

The typical description of ACPO is an elderly patient who has multiple underlying medical conditions and may have re- cently undergone abdominal or nonabdominal surgery. Orthopedic surgery is commonly associated with ACPO and a healthy clinical suspicion in such patients can often lead to early diagnosis and treatment. ${ }^{8}$ Symptoms of abdominal pain, distention, nausea, and vomiting are common. Inability to pass flatus and stool are common but not invariably present, and the clinician should, therefore, be wary of discounting this diagnosis in a patient who continues to have bowel function, including diarrhea, which may occur because of hypersecretion of water. ${ }^{9}$ Fever has been noted in $78 \%$ of the patients with ischemic or perforated bowel, although it may also be found in $31 \%$ of the patients with viable bowel as well. ${ }^{9}$ Significant abdominal distention with tympany is invariably seen on physical examination, and auscultation often reveals high-pitched, but sometimes tinkling bowel sounds, however, bowel sounds may be absent altogether. Abdominal tenderness should increase concern for ischemic bowel or perforation.

\section{Diagnosis}

Diagnosis of ACPO is one of exclusion. It is essential to rule out any mechanical obstruction before adopting and initiating a plan for the treatment of ACPO. The clinical presentation has been discussed, thus when having a patient who presents with signs and symptoms of ACPO, several diagnostic modalities should be considered to determine this diagnosis.

Laboratory values are rarely diagnostic, although correction of electrolyte imbalance may be helpful in the treatment of the condition, and an elevated white blood cell count, lactate, or C-reactive protein may be a cause for concern of more advanced perforated or ischemic bowel. The patient's medical record should be reviewed for any recent or past colonoscopies. This can help to assure that no other colonic pathology exists. Diagnostic colonoscopy is not advised and usually not helpful in the setting of an unprepped bowel for determining whether the patient has a mechanical obstruction.

An initial radiographic abdominal series may help identify an obstructive picture and rule out anatomic sources of obstruction, such as a cecal or sigmoid volvulus or other obstructing lesion. In addition, perforation may be 
A
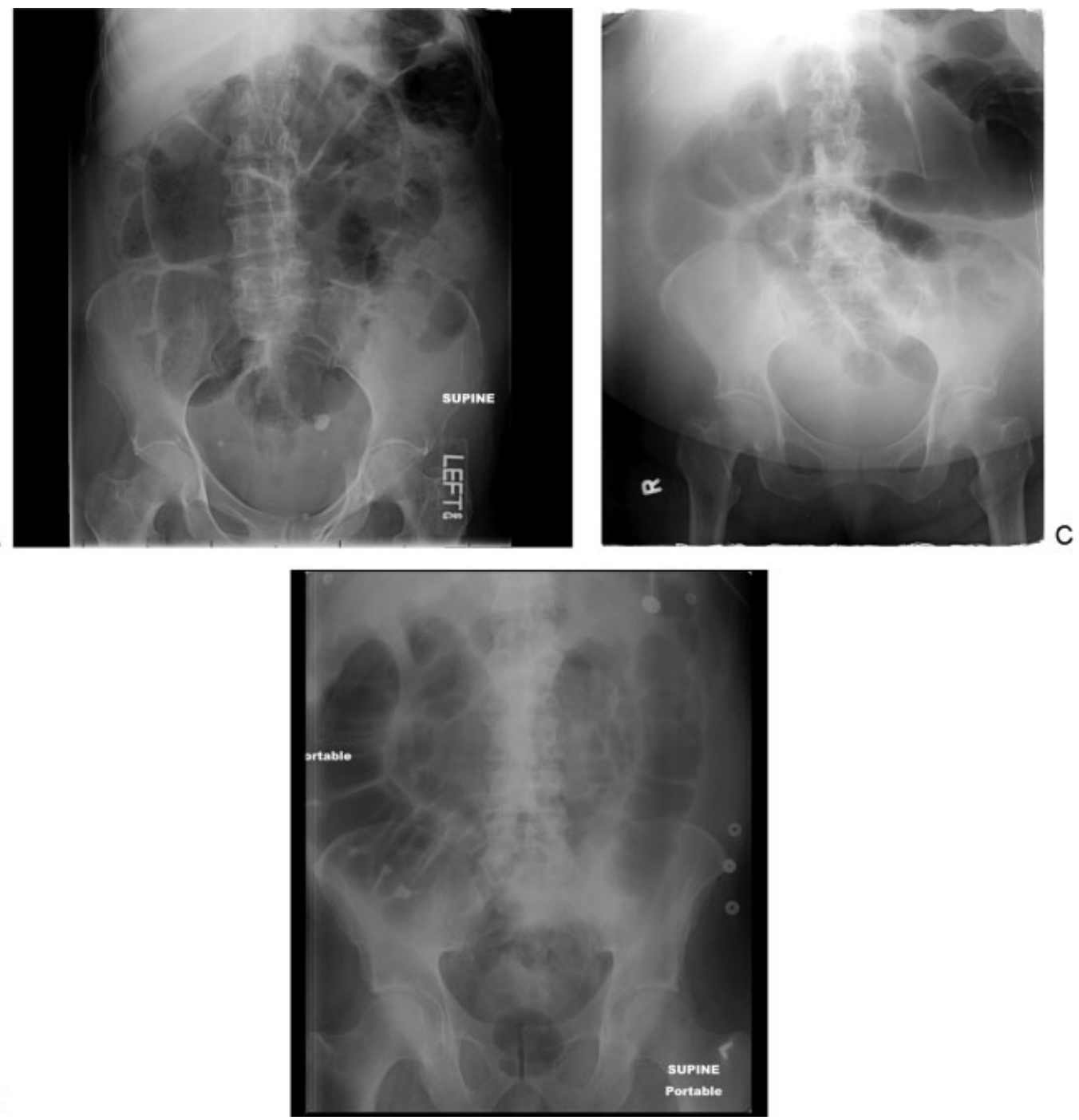

B

Fig. 1 Abdominal X-rays of two elderly women admitted to the hospital for abdominal distension. (A, B) Distension is pancolonic and extends to the rectum, suggestive of acute colonic pseudoobstruction (APCO). (C) Paucity of air in the sigmoid or rectum suggests mechanical large bowel obstruction rather than ACPO.

appreciated if present, and small bowel obstruction may be ruled out. Finally, colonic, or more specifically, cecal diameter should be measured. A cecal dilation of 9 to $12 \mathrm{~cm}$ is suggested to cause concern for impending perforation. ${ }^{10}$ - Fig. $1 \mathbf{a}$ and $\mathbf{1 b}$ demonstrate the radiographic findings in a plain film of patients with ACPO. - Fig. 1c demonstrates the bowel gas pattern of a patient with a mechanical large bowel obstruction. Paucity of gas in the rectum may be a distinguishing clue to differentiate one from another.

A computed tomography (CT) scan with oral, intravenous, and rectal contrast is essential in assuring there is no source of mechanical obstruction or underlying gastrointestinal pathology or neoplasm. In an elderly patient or patients with poor renal function, intravenous contrast should be used with caution or avoided altogether. Additional caution must be exercised with the administration of rectal contrast as there is a risk of perforation in the setting of extreme colonic dilation. A water soluble agent, such as gastrografin, should be used over barium in case a leak or perforation should occur. ${ }^{11-14}$
A typical radiographic finding of ACPO on CT scan is proximal colonic dilation with a transition point at the level of the splenic flexure. ${ }^{10-14}$

\section{Treatment}

\section{Conservative Measures and Medical Therapy}

The treatment strategy for ACPO is initially medical in nature with good success rates. ${ }^{15}$ If medical treatment fails, then procedurally based endoscopic therapy, followed ultimately by surgical intervention may be necessary.

After the diagnosis of ACPO has been made by ruling out a mechanical colonic obstruction, treatment begins with conservative medical therapy. The complete history, physical examination, and the patient's pharmacologic and medical care need to be thoroughly reviewed. It is essential to understand the patient's medical condition. One needs to know the patient's ambulatory status and history of any recent procedures or surgeries. As long as the patient is stable, has no 
Radiographically assure patient has a non-mechanical bowel obstruction

- Obtain and review CT scan

- Review prior colonscopies if available

$\downarrow$

Begin conservative measures

- Correct electrolytes and fluid balance

- Stop all opiates

- Stop all constipating/antimotility medications

- NG tube decompression

- Encourage ambulation

\section{$\downarrow$}

May continue conservative measures for up to $72 \mathrm{~h}$ if

- Cecal diameter $<12 \mathrm{~cm}$

- No peritoneal signs

$\downarrow$

If conservative treatment fails

- Neostigmine (2 mg IV over 2-5 min) in cardiac monitored setting (have atropine available)

$\downarrow$

Colonic decompression +/- placement of a rectal tube

\section{$\downarrow$}

Surgical placement of cecostomy tube versus subtotal colectomy

Fig. 2 An evidence-based treatment algorithm for acute colonic pseudoobstruction.

peritoneal signs, and the cecal diameter is less than $12 \mathrm{~cm}$ on radiographic imaging, conservative measures are initiated. Conservative measures involve correcting electrolyte abnormalities, discontinuing opiates, nasogastric tube decompression, correcting any fluid imbalance, cessation of any antimotility agents, and discontinuing oral intake. Furthermore, attempts should be made to increase patient mobility if possible. These measures may be continued for up to 48 to 72 hours as long as the patient remains stable and has no peritoneal signs or increase in cecal diameter to $12 \mathrm{~cm}$. This conservative approach is associated with an overall mortality rate of $14 \%$ and a success rate as high as $70 \%{ }^{16}$

In a stable, but unimproved patient, the next step in treatment of ACPO has changed in recent years. The current recommendation now is to proceed with pharmacologic intervention with neostigmine in a cardiac-monitored setting. ${ }^{17}$ Baseline electrocardiogram should be obtained and a review of any cardiac history should be undertaken. Neostigmine is a parasympathomimetic which acts by inhibiting acetylcholinesterase. This allows for increased acetylcholine availability and direct parasympathetic stimulation by nicotinic and muscarinic receptors. ${ }^{18}$ The dosage of neostigmine is $2 \mathrm{mg}(\mathrm{mg})$ intravenously (IV) over 1 to 60 minutes (generally $2-5 \mathrm{mi}-$ nutes). This typically causes a large parasympathetic stimulation resulting in high amplitude colonic peristalsis and subsequent flatus and/or bowel movement. It should be noted that the parasympathetic stimulation may cause a profound bradycardia, thus the need for a monitored cardiac setting. Also, subsequent abdominal discomfort, emesis, and excessive salivation may occur. Oftentimes, a step-down unit or postsurgical unit may be used for this situation. Atropine ( $0.5 \mathrm{mg}$ IV) must be available for bradycardia if it occurs. Neostigmine provided a $91 \%$ initial clinical response in a double-blinded study compared with placebo. ${ }^{14}$ If no initial response is seen in the first 3 hours, neostigmine $2 \mathrm{mg}$ IV may be repeated every 3 hours for up to three total doses ( $\mathbf{- F i g . ~ 2 ) . 1 5 , 1 9 - 2 1}$

Once there is resolution of the ACPO, it is suggested that a polyethylene glycol (PEG) solution should be started to prevent recurrence. In a randomized study after successful pharmacologic decompression with neostigmine, there were no recurrences in patients receiving PEG therapy versus $33 \%$ recurrence in the placebo group. ${ }^{22}$ Additional forms of pharmacologic therapy have been attempted after failure of parasympathomimetics, including NO synthase inhibitors, motilin-receptor agonists, and opioid-receptor antagonists, which affect postoperative ileus. However, definitive efficacy for ACPO has not been shown with other pharmacologic choices and neostigmine has become the pharmacologic standard. ${ }^{23,24}$

\section{Endoscopic Therapy}

For those patients who fail pharmacologic therapy, the next step is to attempt endoscopic colonic decompression in conjunction with placement of a nontraumatic large diameter soft catheter rectal tube.

Alternatively, sustained decompression can be achieved by placing a long catheter into the cecum under fluoroscopic guidance or endoscopic piggyback.

Harig et al and Messmer et al suggest that fluoroscopic catheterization of the cecum can be achieved using the following procedural steps: (1) Use of a colonoscope with minimum to no insufflation, while suctioning the distension until the cecum is reached, (2) placement of a guidewire through the biopsy channel of the colonoscope, (3) removal of the colonoscope while leaving the guidewire in place, (4) under fluoroscopic guidance, a catheter can be threaded over the guidewire into the cecum, and (5) secured with tape or suture to the inner thigh of the patient. ${ }^{24,26}$

Stephenson et al suggest that bowel decompression can be achieved without the aid of fluoroscopy if a long intestinal tube is piggybacked on the colonoscope as it is placed in the cecum. ${ }^{27}$ The advantage of this technique is that an abdominal radiograph is sufficient to confirm tube placement, thus 
eliminating the need for fluoroscopy. Groff ${ }^{28}$ describes his method of decompression as follows: (1) Use a two channel colonoscope, one that allows for both suction and insufflation. (2) Fill the biopsy channel of the colonoscope with biopsy forceps and pass the forceps through the channel. (3) Place silk sutures through the holes at the tip of any appropriate suction tube. (4) Grasp the silk sutures with the biopsy forceps and pull the suture partially into the biopsy channel of the colonoscope. The tube will trail behind the tip of the colonoscope. (5) Insert the colonoscope into the anus and advance as far as possible. (6) Advance the biopsy forceps beyond the tip of the colonoscope and release the silk. (7) Confirm placement of tube with X-ray. (8) Place tube to low intermittent suction.

According to a review of the literature by Rex, colonic decompression was associated with an initial success rate of $69 \%$ and a recurrence rate of $40 \%$ when concurrent tube decompression was not performed. ${ }^{29} \mathrm{~A}$ subsequent report by Geller et al noted that a tube placed in the right and transverse colon had clinical success rate of 57 and 33\%, respectively, whereas procedures without tube placement had poor clinical success rate of $25 \%{ }^{30}$ Colonoscopic decompression is associated with a morbidity and mortality of 1.7 and $3.4 \%$, respectively, including perforation. ${ }^{13,14,17,24,29}$ Tsirline et al retrospectively compared colonoscopic decompression with neostigmine administration for ACPO and noted that of a total of 77 patients, 46 had one colonoscopy and 6 had two colonoscopies, while 22 patients had one neostigmine administration, 14 had two doses, and 8 patients had multiple doses of neostigmine. Clinical response after one colonoscopy was significantly more effective than either 1 or 2 doses of neostigmine ( 75 vs. 35 or $56 \%$ ). ${ }^{31}$ It should be noted, however, that colonoscopy requires specific expertise, higher cost, and more time than neostigmine administration.

\section{Tube Cecostomy}

As described by Nishiwaki et al, percutaneous cecostomy tube placement under ultrasound and fluoroscopic guidance involves the following steps: (1) Under ultrasound guidance, anchor the anterior wall of the cecum to the abdominal wall at three points with T-fastener fixation devices (Easy Tie; Boston Scientific, Japan KK., Tokyo, Japan). (2) Puncture cecum with 16-gauge needle, insert a guidewire through the outer sheath, and use Seldinger technique under fluoroscopic guidance to dilate the colocutaneous fistula up to 24 French. (3) Percutaneously place a 24 French cecostomy tube (Securi-T; Boston Scientific, Natick Mass) in the cecum. (4) Administer cefoperazone intramuscularly or IV for 3 days as aprophylactic antibiotic. ${ }^{32}$

Tube cecostomy has reported success rates of up to $100 \%$ when required. ${ }^{9,33-35}$

However, surgically placed tube cecostomy has a reported morbidity and mortality of 50 and $19 \%$, respectively. Potential complications include pressure necrosis, granulation tissue formation, cellulitis of the abdominal wall, ventral hernia, persistent colocutaneous fistula, tube occlusion, and sepsis. Thus, many have advocated for attempting a percutaneous (radiographically) or percutaneous endoscopic cecostomy
(PEC) tube placement. ${ }^{33}$ This avoids the use of general anesthesia and is placed under the guidance of colonoscopic transillumination of the cecum. Air is vented and the tube is ideally removed once the condition and underlying cause have resolved. Direct comparison of PEC to other methods of colonoscopic decompression is lacking.

\section{Surgical Therapy}

If conservative medical, pharmacologic, and endoscopic measures fail to achieve resolution of ACPO, then surgical procedures may be necessary. These include percutaneous, percutaneous endoscopic or surgical cecostomy tube placement or subtotal colectomy. Surgical procedures carry higher morbidity and mortality. Nonetheless, in the setting of failure of prior therapy, clinical deterioration, peritonitis, or cecal diameter $12 \mathrm{~cm}$ or greater, surgical therapy may be warranted. Surgical mortality for ACPO has been reported to be as high as 30 to $44 \%$, especially in patients with ischemic or perforated bowel and advanced age. ${ }^{9,16,34}$ Operative interventions may include ileostomy or colostomy, bowel resection, exteriorization, cecostomy tube placement, intraoperative long colon tube placement, or other procedures. Of these operations, resection or exteriorization/ cecostomy have the highest success rate with the lowest morbidity and are the procedures of choice when needed. ${ }^{9}$

\section{Methods of Prevention}

The data for prevention of ACPO are limited and most individual guidelines favor similar modalities used for prevention of ileus and constipation: limiting opiates, early ambulation, laxatives in the setting of narcotic use, electrolyte and fluid correction. Currently, there are no randomized controlled trials looking at prevention of ACPO.

\section{Conclusion}

ACPO is a potentially morbid and even fatal condition commonly concerning already unwell, elderly patients. Medical management is generally successful, and it is considerably preferable over more dangerous surgical therapies. When conservative measures fail, neostigmine is the treatment of choice and it has a high success rate when administered properly. Careful attention to prevention is warranted in all patients at risk.

\section{Acknowledgments}

The authors thank Allison Estep and Anne Tretin from Georgetown University School of Medicine and Alexandra Dubinskaya, MD, volunteer research assistant at MedStar Washington Hospital Center for their editorial assistance. The ASCRS CREST Committee has permitted us to share our work in this manner.

\section{References}

1 Bode WE, Beart RW Jr, Spencer RJ, Culp CE, Wolff BG, Taylor BM. Colonoscopic decompression for acute pseudoobstruction of the colon (Ogilvie's syndrome). Report of 22 cases and review of the literature. Am J Surg 1984;147(2):243-245 
2 Wegener M, Börsch G. Acute colonic pseudo-obstruction (Ogilvie's syndrome). Presentation of 14 of our own cases and analysis of 1027 cases reported in the literature. Surg Endosc 1987;1(3): 169-174

3 Ogilvie H. Large-intestine colic due to sympathetic deprivation; a new clinical syndrome. BMJ 1948;2(4579):671-673

4 Barrett KE. Chapter 9. Intestinal motility. In: Barrett KE, eds. Gastrointestinal Physiology, 2nd ed. New York, NY: McGraw-Hill; 2014

5 Kimoto M, Zeredo JL, Ota MS, Nihei Z, Toda K. Comparison of stress-induced modulation of smooth-muscle activity between ileum and colon in male rats. Auton Neurosci 2014;183:8-11

6 Onori L, Aggio A, D'Alo' S, et al. Role of nitric oxide in the impairment of circular muscle contractility of distended, uninflamed mid-colon in TNBS-induced acute distal colitis in rats World J Gastroenterol 2005;11(36):5677-5684

7 Kodama Y, Iino S, Shigemasa Y, Suzuki H. Properties of acetylcholine-induced relaxation of smooth muscle isolated from the proximal colon of the guinea-pig. J Smooth Muscle Res 2010; 46(4):185-200

8 Norwood MG, Lykostratis H, Garcea G, Berry DP. Acute colonic pseudo-obstruction following major orthopaedic surgery. Colorectal Dis 2005;7(5):496-499

9 Vanek VW, Al-Salti M. Acute pseudo-obstruction of the colon (Ogilvie's syndrome). An analysis of 400 cases. Dis Colon Rectum 1986;29(3):203-210

10 Davis L, Lowman RM. An evaluation of cecal size in impending perforation of the cecum. Surg Gynecol Obstet 1956;103(6): 711-718

11 Choi JS, Lim JS, Kim H, et al. Colonic pseudoobstruction: CT findings. AJR Am J Roentgenol 2008;190(6):1521-1526

12 Jacob SE, Lee SH, Hill J. The demise of the instant/unprepared contrast enema in large bowel obstruction. Colorectal Dis 2008; 10(7):729-731

13 Beck D. ASCRS Textbook of Colon and Rectal Surgery. 2nd ed. New York: Springer; 2011

14 Gordon P, Nivatvongs S. The Principles and Practice of Surgery for the Colon, Rectum, and Anus. 3rd ed. New York: CRC Press; 2007

15 Ponec RJ, Saunders MD, Kimmey MBN. Neostigmine for the treatment of acute colonic pseudo-obstruction. N Engl J Med 1999;341(3):137-141

16 De Giorgio R, Barbara G, Stanghellini V, et al. Review article: the pharmacological treatment of acute colonic pseudo-obstruction. Aliment Pharmacol Ther 2001;15(11):1717-1727

17 Trevisani GT, Hyman NH, Church JM. Neostigmine: safe and effective treatment for acute colonic pseudo-obstruction. Dis Colon Rectum 2000;43(5):599-603

18 Tangsucharit P, Takatori S, Sun P, et al. Do cholinergic nerves innervating rat mesenteric arteries regulate vascular tone? Am J Physiol Regul Integr Comp Physiol 2012;303(11):R1147-R1156
19 White L, Sandhu G. Continuous neostigmine infusion versus bolus neostigmine in refractory Ogilvie syndrome. Am J Emerg Med 2011;29(5):576.e1-576.e3

20 Paran H, Silverberg D, Mayo A, Shwartz I, Neufeld D, Freund U. Treatment of acute colonic pseudo-obstruction with neostigmine. J Am Coll Surg 2000;190(3):315-318

21 Turégano-Fuentes F, Muñoz-Jiménez F, Del Valle-Hernández E, et al. Early resolution of Ogilvie's syndrome with intravenous neostigmine: a simple, effective treatment. Dis Colon Rectum 1997;40(11):1353-1357

22 Sgouros SN, Vlachogiannakos J, Vassiliadis K, et al. Effect of polyethylene glycol electrolyte balanced solution on patients with acute colonic pseudo obstruction after resolution of colonic dilation: a prospective, randomised, placebo controlled trial. Gut 2006;55(5):638-642

23 Borgaonkar MR, Lumb B. Acute on chronic intestinal pseudoobstruction responds to neostigmine. Dig Dis Sci 2000;45(8): 1644-1647

24 Harig JM, Fumo DE, Loo FD, et al. Treatment of acute nontoxic megacolon during colonoscopy: tube placement versus simple decompression. Gastrointest Endosc 1988;34(1):23-27

25 Harrison ME, Anderson MA, Appalaneni V, et al; ASGE Standards of Practice Committee. The role of endoscopy in the management of patients with known and suspected colonic obstruction and pseudo-obstruction. Gastrointest Endosc 2010;71(4):669-679

26 Messmer JM, Wolper JC, Loewe CJ. Endoscopic-assisted tube placement for decompression of acute colonic pseudo-obstruction. Endoscopy 1984;16(4):135-136

27 Stephenson KR, Rodriguez-Bigas MA. Decompression of the large intestine in Ogilvie's syndrome by a colonoscopically placed long intestinal tube. Surg Endosc 1994;8(2):116-117

28 Groff W. Colonoscopic decompression and intubation of the cecum for Ogilvie's syndrome. Dis Colon Rectum 1983;26(8):503-506

29 Rex DK. Acute colonic pseudo-obstruction (Ogilvie's syndrome). Gastroenterologist 1994;2(3):233-238

30 Geller A, Petersen BT, Gostout CJ. Endoscopic decompression for acute colonic pseudo-obstruction. Gastrointest Endosc 1996; 44(2):144-150

31 Tsirline VB, Zemlyak AY, Avery MJ, et al. Colonoscopy is superior to neostigmine in the treatment of Ogilvie's syndrome. Am J Surg 2012;204(6):849-855, discussion 855

32 Nishiwaki S, Hatakeyama H, Takada J, et al. Transcecostomal colonic stent placement after US-guided percutaneous cecostomy. Gastrointest Endosc 2010;71(6):1097-1099

33 Benacci JC, Wolff BG. Cecostomy. Therapeutic indications and results. Dis Colon Rectum 1995;38(5):530-534

34 vanSonnenberg E, Varney RR, Casola G, et al. Percutaneous cecostomy for Ogilvie syndrome: laboratory observations and clinical experience. Radiology 1990;175(3):679-682

35 Ramage JI Jr, Baron TH. Percutaneous endoscopic cecostomy: a case series. Gastrointest Endosc 2003;57(6):752-755 